\title{
Tissue and circulating immunoreactive protein for MMP-2 and TIMP-2 in head and neck squamous cell carcinoma-tissue immunoreactivity predicts aggressive clinical course
}

\author{
Henni Ruokolainen ${ }^{1}$, Paavo Pääkkö ${ }^{2}$ and Taina Turpeenniemi-Hujanen ${ }^{1}$ \\ ${ }^{1}$ Department of Oncology and Radiotherapy, University of Oulu and Oulu University Hospital, Oulu, Finland \\ and ${ }^{2}$ Department of Pathology, University of Oulu and Oulu University Hospital, Oulu, Finland
}

\begin{abstract}
Useful markers showing biological aggressiveness of head and neck squamous cell carcinoma (HNSCC) are needed to predict the outcome of the disease. MMP-2 is associated with aggressive behavior of several solid cancers. In this study, the clinical significance of tumor tissue and circulating immunoreactive proteins for MMP-2 and TIMP-2 was assessed in HNSCC. The study group consisted of 74 patients with HNSCC and 44 healthy controls. Expression of MMP-2 and TIMP-2 was examined in paraffin-embedded tumor sections by immunohistochemical methods using specific antibodies. The pretreatment serum levels of MMP-2, TIMP-2 and MMP-2:TIMP-2 complex were quantitatively measured by ELISA assay. The results were compared with the clinicopathological factors of the disease and the patients' outcome. Immunohistochemical overexpression of MMP-2 in tumor was found to be prognostic for shortened survival in HNSCC, the 5-year cumulative relapse-free survival being $42 \%$ in patients with high positivity for MMP-2 in tumor vs $61 \%$ in cases with a negative or only weakly MMP-2-positive tumor $(P=0.045)$. Tissue MMP-2 positivity was also strongly connected with later lymph node or hematogenic relapses and associated to the cause-specific survival $(P=0.055)$. Similarly, the 5 -year cause-specific survival was significantly poorer in patients with extensive positive immunostaining for tumor TIMP-2 than in those with a TIMP-2-negative tumor (40 vs $64 \%, P=0.038)$. Patients with a TIMP-2-positive tumor also had an unfavorable 5-year relapse-free survival rate (43 vs $60 \%$, respectively, $P=0.071)$. Additionally, the overexpression of TIMP-2 was a powerful predictor of later lymph node or hematogenous metastases in HNSCC. Serum levels of MMP-2, TIMP-2 or MMP-2:TIMP-2 complex failed to associate with the clinical behavior of HNSCC in this material. The results of this study provide evidence that MMP-2 and TIMP-2 immunoreactive protein in tumor tissue of HNSCC patients, but not when assayed from preoperative serum samples, are prognostic in estimation of the aggressive clinical course of HNSCC.
\end{abstract}

Modern Pathology (2006) 19, 208-217. doi:10.1038/modpathol.3800506; published online 4 November 2005

Keywords: MMP-2; TIMP-2; head and neck squamous cell carcinoma; metastasis; prognostic marker; causespecific survival; relapse-free survival

Head and neck cancers consist of a heterogenous group of neoplasias, $90 \%$ of which are squamous cell carcinomas (head and neck squamous cell carcinoma (HNSCC)). The occurrence of HNSCC correlates most closely with the use of tobacco as well as with alcohol consumption. ${ }^{1}$ The most

Correspondence: Dr H Ruokolainen, MD, Department of Oncology and Radiotherapy, Oulu University Hospital, PO Box 22, Oulu FIN-90211, Finland.

E-mail: hruokola@paju.oulu.fi

Received 6 July 2005; revised 7 September 2005; accepted 24 September 2005; published online 4 November 2005 commonly used indicators include the clinical stage of the disease at the time of diagnosis, especially the number of lymph node metastases. ${ }^{2}$ New prognostic markers have been searched in order to better identify patient groups in need of more aggressive treatments. Although experimental studies have discovered some biological markers, only some of them have so far been tested as clinical markers in HNSCC and none of them are accepted widely as parameters in clinical decision making.

Matrix metalloproteinases are a family of zinc- or calcium-dependent endopeptidases. These enzymes are associated with proteolytic degradation of the 
extracellular matrix. ${ }^{3}$ The role of matrix metalloproteinases in tumor invasion and metastatic process has been confirmed. The degradation of ECM by matrix metalloproteinases is a critical process in the progression of malignant tumors, because degradation of ECM is required in tumor angiogenesis, as well as in tumor invasion and metastasis. Gelatinases are thought to be key enzymes as they degrade some main components of ECM. ${ }^{4-6}$ Matrix metalloproteinases are secreted as latent proenzymes. Their secretion is regulated at transcriptional level and their proteolytic activity is further controlled by activation and inhibition.

Tissue inhibitors of matrix metalloproteinases (TIMPs) are known to have various physiological functions. They are capable of inhibiting the activity of MMPs and they can form complexes with either latent or activated enzymes. In addition to their inhibitory role, TIMPs can also take part in the activation of MMPs. ${ }^{7}$ The TIMPs also seem to have antiangiogenic activity and they are able to act as growth factors. ${ }^{8}$ It is fairly uncertain whether TIMPs have a protecting role in cancer cell growth and invasion or whether they contribute to the process of formation of metastases.

There is a growing amount of clinical data showing that expression of gelatinases as well as tissue inhibitors of gelatinases is associated with clinical course in some solid cancers. Overexpression of cancer tissue MMP-2 has been linked with tumor invasion, metastasis and poor survival in many tumor types including some preliminary studies in HNSCC. ${ }^{9-18}$ Only few studies have tried to evaluate whether TIMP-2 could serve as a prognostic indicator for survival in HNSCC. ${ }^{18}$ We have previously demonstrated that MMP-9 and TIMP-1 immunoreactive proteins in both tumor tissue and serum are associated with shortened relapse-free and cause-specific survival in HNSCC patients. ${ }^{19-21}$ In this study, the expression of MMP-2 and TIMP-2 in tumor tissue was studied and compared to the circulating level of these markers in HNSCC patients to explore their role in the estimation of prognosis of HNSCC patients.

\section{Materials and methods}

\section{Patients}

The study series included 74 patients referred to Oulu University Hospital for treatment of a primary squamous cell head and neck carcinoma between the years 1994 and 1996. The patients were recruited consecutively to the study and they were followed for a minimum of 5 years. Serum samples were taken during the first visit to the hospital before the therapy. Serum samples collected in tubes with no additives were stored frozen in $-70^{\circ} \mathrm{C}$ until used for the study. Immunostaining of the paraffinembedded tissue samples was performed for cases where a representative amount of tissue was avail- able, in 72 cases for MMP-2 and 70 cases for TIMP-2. There were pretreatment serum samples from 68 cases for TIMP-2 and MMP-2:TIMP-2 complex ELISA analyses, whereas the number of available serum samples for MMP-2 analyses was 65. The study protocol was approved by the Ethical Committee of Oulu University in March 1994, and renewed by the Ethical Committee of Oulu University Hospital in July, 2002. For control values, serum samples were taken from 44 healthy volunteers (27 female, 17 male), without a history of a known malignant disease or recent surgery.

The mean age of the patients was 64 years (minimum 28 years and maximum 88 years). The stage of the disease, tumor size and lymph node involvement was determined according to the International Union Against Cancer TNM classification. ${ }^{22}$ The histological grade of the tumors was reviewed and classified according to the World Health Organization Classification of head and neck tumors. ${ }^{23}$

The treatment was carried out according to the local protocol according to the stage of the tumor. A total of 23 patients were radically operated and received $50-60 \mathrm{~Gy}$ postoperative radiotherapy. In all, 15 patients received preoperative radiotherapy $(50 \mathrm{~Gy})$ treatment before the surgical operation. A total of 18 were treated only by a radical surgical operation, and nine received only radiotherapy (50$64 \mathrm{~Gy})$. No patients received any adjuvant chemotherapy. Two of the patients had an advanced carcinoma and received only palliative treatment.

\section{ELISA Assays for MMP-2, TIMP-2 and MMP-2:TIMP-2 Complex}

ELISA assays were performed on eight-well EIA microtiter plates (Corning, NY, USA) using the standard protocols. ${ }^{24}$ Polyclonal chicken antibody was used as a second antibody. OPD solution (P-1526, Sigma, Germany) was used to visualize the peroxidase label. The color formation was measured on $492 \mathrm{~nm}$. Between each step of the procedure the wells were thoroughly washed with $0.05 \%$ Tween-phosphate-buffered saline. The sensitivity of the assays was $0.37 \mathrm{ng} / \mathrm{ml}$ for MMP-2, $2 \mathrm{ng} / \mathrm{ml}$ for TIMP-2 and $2 \mathrm{ng} / \mathrm{ml}$ for the MMP-2:TIMP-2 complex. The detection of the samples took place on the linear area of the standard curve of each analysis.

For the quantification of the MMP-2:TIMP-2 complex, the plate was coated with a commercial monoclonal anti-TIMP-2 antibody (code T2-101; SBA Sciences, Oulu, Finland). The bound complex was detected with a polyclonal anti-MMP-2 antibody (code DB-202; SBA Sciences, Oulu, Finland). When measuring the total TIMP-2 (free and complexed with MMP-2), monoclonal anti-TIMP-2 antibody was used (code T2-101; SBA Sciences; Oulu, Finland) for coating the microtiter plates before 
adding the samples and standards and the bound proteins were detected with a polyclonal anti-TIMP2 antibody (code DB-205; SBA Sciences, Oulu, Finland) as described above. Serum MMP-2 concentration was determined by using human MMP-2 ELISA (code RPN2617, Amersham) according to the manufacturer's instructions. This assay recognizes the precursor of MMP-2 (proMMP-2), that is, free pro-MMP-2 and that complexed with TIMP-2, but not the active form of MMP-2.

\section{Immunohistochemical Staining of MMP-2 and TIMP-2 and Evaluation of the Immunostaining}

Paraffin-embedded sections ( $4 \mu \mathrm{m}$ ) from the primary tumors of head and neck carcinomas were stained using the avidin-biotin-immunoperoxidase technique. Paraffin sections were incubated at $37^{\circ} \mathrm{C}$ for at least $4 \mathrm{~h}$, dewaxed (Histo-Clear ${ }^{\mathbb{B}}$, National Diagnostic, Atlanta, GA, USA) and hydrated. For MMP-2 immunostaining the slides were treated with $0.4 \%$ pepsin (Sigma, St Louis, MO, USA) at $37^{\circ} \mathrm{C}$ for $20 \mathrm{~min}$. Endogenous peroxidase activity was blocked by incubating the slides in 3\% hydrogen peroxidase/methanol for $10 \mathrm{~min}$, and nonspecific binding was blocked with $10 \%$ goat serum for 15 min. A mouse monoclonal antibody for MMP-2, $7.2 \mu \mathrm{g} / \mathrm{ml}$ (CA-4001; Diabor Ltd, Oulu, Finland) and TIMP-2, $20.0 \mu \mathrm{g} / \mathrm{ml}$ (R\&D Systems, Minneapolis, MN, USA) were used as primary antibodies. The primary antibodies in $0.01 \mathrm{M}$ phosphate buffer, $0.9 \% \mathrm{NaCl}(\mathrm{pH}$ 7.5) were mixed with Antibody Diluent $^{\circledR}$ (Dako, Glostrup, Denmark) in TIMP-2 stainings and in MMP-2 stainings the antibodies were mixed with $1 \%$ bovine serum albumin. The specimens were incubated with primary antibody for $60 \mathrm{~min}$ at room temperature in a humidified atmosphere in MMP-2 staining, and the incubation time for specimens in TIMP-2 staining was $12 \mathrm{~h}$. The immunohistochemical staining was continued according to the manufacturer's protocol using Histostain-bulk kit ${ }^{\circledR}$ (Zymed, San Francisco, CA, USA) that uses a biotinylated antimouse IgG as a second antibody and a streptavidin conjugate as a linkage protein. The final reaction was visualized by using an aminoethyl carbazol substrate kit (AEC$\mathrm{kit}^{\mathbb{R}}$, Zymed, San Francisco, CA, USA); in TIMP-2 immunostaining the antibody reaction was visualized by using a fresh substrate solution containing romulin aminoethyl carbazol (Romulin AEC-Chromogen, Biocare Medical, Walnut Creek, CA, USA) and the sections were counterstained with hematoxylin, dehydrated and mounted glycerol-vinyl-alcohol (GVA mount ${ }^{\mathbb{R}}$; Zymed) in MMP-2 stainings and (Histomount $^{\mathbb{R}}$, National Diagnostics, New Jersey, USA) with ksylen in the TIMP-2 stainings. Each set of staining always included a separate known positive control and a negative control, where the primary antibody for MMP-2 or TIMP-2 was replaced with mouse nonimmuno IgG.
The slides were analyzed by two independent observers blinded from clinical data and the immunoreactivity in the malignant cells in each section was graded from 0 to 3 according to the extent of positive staining. The case was considered positive when $>1 \%$ of the tumor cells showed a positive staining. Weak positivity was marked as $+(25 \%$ or less of the tumor cells staining positive $)$ and moderate positivity was marked as $++(26-50 \%$ of tumor cells staining positive). Cases showing a positive staining in more than $50 \%$ of the tumor cells were considered to be extensively positive for MMP-2 or TIMP-2 $(+++)$.

\section{Statistical Analysis}

All statistical analyses were performed by using the SPSS software system (SPSS for Windows, version 11.0, Chicago, IL, USA). The correlations of tumor stage, TNM classification, histological grade and the primary anatomical site were analyzed separately according to the MMP-2 or TIMP-2 immunoreactivity and to the corresponding immunoreactive protein levels measured by ELISA. The statistical significance of these correlations was determined with Fisher's exact test. To compare the difference of the means between groups, the Mann-Whitney $U$ test was used. The cause-specific and relapse-free survival rates were analyzed using the Kaplan-Meier method, and the statistical differences in survival among subgroups were compared by a log-rank and Breslow test. ${ }^{25}$ The cause-specific survival was defined as the time from the date of diagnosis to the date of death due to head and neck carcinoma. The cases were censored on the date of the last control or at the time of death to another disease. Similarly, the relapse-free survival was calculated from the date of diagnosis to the date of relapse, and the case was censored at the time of the last followup visit.

\section{Results}

Patterns of Expression of the Immunoreactive Protein of MMP-2 and TIMP-2 in Squamous Cell Carcinoma of Head and Neck

Paraffin-embedded tissue sections were available for MMP-2 immunostaining in 72 cases. Of 72 primary HNSCC tumors, $64(89 \%)$ showed positive staining of MMP-2 immunoreactive protein. In $48(67 \%)$ cases, the staining was moderate or extensive (more than $25 \%$ of tumor cells positive) (Table 1), whereas weak positivity was observed in 16/72 (22\%) patients $(+; 25 \%$ or less of the tumor cells staining positive). Overexpression of the TIMP-2 immunoreactive protein was found in $46(66 \%)$ of the 70 cases (Table 1). Extensive positive staining for TIMP-2 $(+++;>50 \%$ of the tumor cells appearing as positive) was found in 10 out of 70 cases 
Table 1 Tissue MMP-2 and TIMP-2 in different subgroups of head and neck carcinoma

\begin{tabular}{|c|c|c|c|c|c|c|}
\hline Patient characteristics & $\mathrm{n}$ & $M M P-2++/+++n(\%)$ & $\mathrm{P}$ & $\mathrm{n}$ & $T I M P-2+/++/+++n(\%)$ & $\mathrm{P}$ \\
\hline All patients & 74 & & & & & \\
\hline Subsets & 72 & $48(67)$ & & 70 & $46(66)$ & \\
\hline \multicolumn{7}{|l|}{ Sex } \\
\hline Male & 48 & $34(71)$ & 0.30 & 48 & $32(67)$ & 0.31 \\
\hline Female & 24 & $14(58)$ & & 22 & $14(64)$ & \\
\hline \multicolumn{7}{|l|}{ Age (years) } \\
\hline $28-50$ & 9 & $6(67)$ & 0.45 & 10 & $8(80)$ & 0.61 \\
\hline $51-65$ & 27 & $20(74)$ & & 27 & $18(67)$ & \\
\hline $66-75$ & 26 & 17 (65) & & 24 & $16(67)$ & \\
\hline $76-88$ & 10 & $8(80)$ & & 9 & $4(45)$ & \\
\hline \multicolumn{7}{|l|}{ Anatomical diagnosis } \\
\hline Oral cavity & 32 & $21(66)$ & 0.61 & 31 & $21(68)$ & 0.78 \\
\hline Larynx & 25 & 17 (68) & & 25 & 17 (68) & \\
\hline Pharynx & 9 & $5(56)$ & & 8 & $4(50)$ & \\
\hline Others & 6 & $5(83)$ & & 4 & $4(100)$ & \\
\hline \multicolumn{7}{|l|}{ Grade } \\
\hline Grade 1 & 15 & $11(73)$ & 0.76 & 14 & $10(71)$ & 0.66 \\
\hline Grade 2 & 45 & 30 (67) & & 46 & $29(63)$ & \\
\hline Grade 3 & 12 & $7(58)$ & & 10 & $7(70)$ & \\
\hline \multicolumn{7}{|l|}{ TNM classification } \\
\hline $\mathrm{T}_{1-2}$ & 38 & $24(63)$ & 0.62 & 37 & $21(57)$ & 0.26 \\
\hline $\mathrm{T}_{3-4}$ & 34 & $24(71)$ & & 33 & $25(76)$ & \\
\hline $\mathrm{N}_{0}$ & 42 & $30(71)$ & 0.33 & 38 & $24(63)$ & 0.71 \\
\hline $\mathrm{N}_{+}$ & 30 & $18(60)$ & & 32 & $22(69)$ & \\
\hline \multicolumn{7}{|l|}{ Stage } \\
\hline I & 10 & $8(80)$ & 0.54 & 10 & $8(80)$ & 0.23 \\
\hline II & 15 & $9(60)$ & & 14 & $6(43)$ & \\
\hline III & 22 & $14(64)$ & & 22 & $14(64)$ & \\
\hline IV & 25 & 17 (68) & & 24 & $18(75)$ & \\
\hline
\end{tabular}

(14\%), moderate positivity $(++; 26-50 \%$ of the tumor cells staining positive) was found in 15 cases $(21 \%)$ and weak positivity $(+; 25 \%$ or less of the tumor cells staining positive) in 21 cases $(30 \%)$. The staining was negative in $34 \%$ $(24 / 70)$ of the cases. The positive immunoreaction was seen as a diffuse staining localized in the cancer cell cytoplasm with both of the antibodies (Figure 1).

\section{Lack of Association of Tumor Immunoreactive Protein of MMP-2 and TIMP-2 with Clinical Prognostic Parameters}

We could not find any correlation between the overexpression of MMP-2 or TIMP-2 immunoreactive proteins and the stage of the disease or with the TNM or histological grade of the tumor. Neither was there any correlation between patients' age or sex or the anatomical site of the tumor and the positive immunoreaction for MMP-2 or TIMP-2 in the primary tumor (Table 1). No statistical differences were found with MMP-2 or TIMP-2 immunohistochemical staining among the patients in different treatment groups.

\section{MMP-2 and TIMP-2 Serum Levels}

The pretreatment serum sample for ELISA analyses was available from 68 patients with the exception of the MMP-2 analysis, where the serum sample was available from 65 patients. The minimum, maximum and mean levels of immunoreactive proteins are listed in Table 2. The serum levels of the MMP-2: TIMP-2 complex were significantly higher in the healthy controls than in HNSCC patients $(P<0.001$, Mann-Whitney test) (Table 2). Also, there was a trend that the mean values of serum MMP-2 immunoreactive protein in healthy controls were higher when compared to those of HNSCC patients. This difference did not quite reach statistical significance $(P=0.09$, Mann-Whitney test). However, no statistical difference was found in TIMP-2 serum levels between HNSCC patients and healthy controls ( $P=0.9$, Mann-Whitney test).

No correlation was observed between serum levels of MMP-2, TIMP-2 and MMP-2:TIMP-2 complex and traditional clinicopathological factors. However, there was a tendency indicating that higher levels of TIMP-2 and MMP-2:TIMP-2 complex were associated with higher age groups in HNSCC patients. We could not detect differences in survival among 

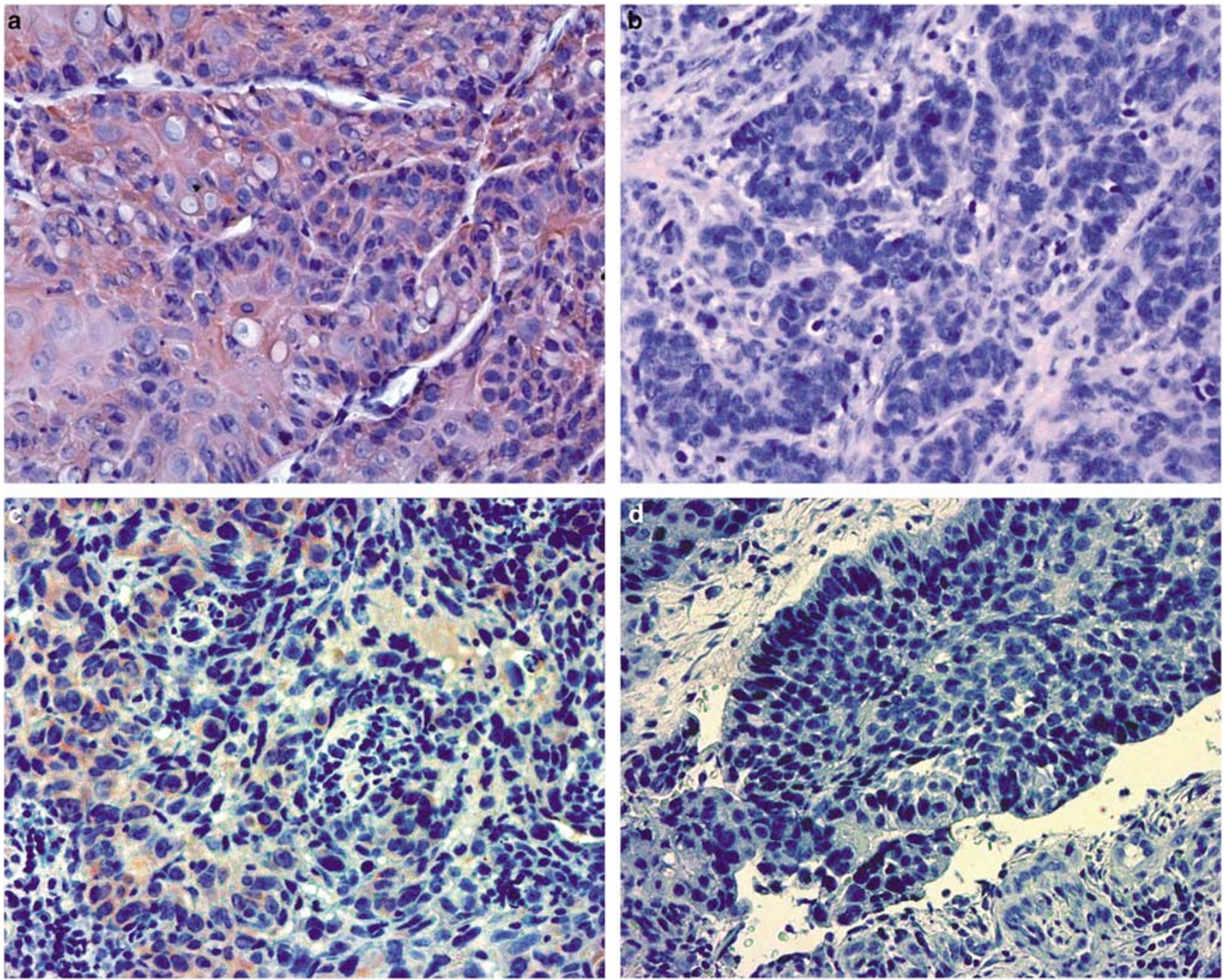

Figure 1 Cytoplasmic immunostaining of matrix metalloproteinase-2 (MMP-2) and tissue inhibitor of matrix metalloproteinase 2 (TIMP-2) in head and neck squamous cell carcinoma. The immunostaining procedure was performed as described using an anti-MMP-2 and anti-TIMP-2 monoclonal antibodies. (a) Tumor with a positive immunostaining for MMP-2. (b) Tumor with a negative immunostaining for MMP-2. (c) Tumor positive for TIMP-2. (d) Tumor negative for TIMP-2.

Table 2 Serum level (ng/ml) of MMP-2 and TIMP-2 immunoreactive protein in head and neck squamous cell carcinoma patients and in healthy controls

\begin{tabular}{lllllc}
\hline & \multicolumn{5}{c}{$n g / m l$} \\
\cline { 2 - 6 } & $\mathrm{n}$ & Min. & Max. & Mean & $\mathrm{P}$ \\
\hline MMP-2 & & & & & \\
HNSCC & 65 & 666 & 2371 & 1312 & 0.09 \\
Control & 44 & 983 & 1939 & 1381 & \\
TIMP-2 & & & & & \\
HNSCC & 68 & 149 & 597 & 323 & 0.9 \\
Control & 44 & 253 & 458 & 321 & \\
MMP-2:TIMP-2 & complex & & & \\
HNSCC & 68 & 214 & 1196 & 532 & $<0.001$ \\
Control & 44 & 465 & 887 & 627 & \\
\hline
\end{tabular}

HNSCC patients when analyzing patients in groups according to different cutoff values of the serum immunoreactive protein levels.

\section{Comparison Between the MMP-2 or TIMP-2 Expression and the Corresponding Circulating Immunoreactive Protein}

There was no significant correlation between the amount of MMP-2 and TIMP-2 immunohistochemical staining. Furthermore, there was no correlation between the tumor expression of TIMP-2 and the serum levels of TIMP-2 immunoreactive protein. Accordingly, no association was found between the TIMP-2 or MMP-2 overexpression in tumor tissue and the serum values of MMP-2 or MMP-2:TIMP-2 complex.

\section{MMP-2 as a Prognostic Factor}

When comparing the relapses (local, lymph node, hematogenous), it was found that the MMP-2 overexpression was significantly higher in the primary tumor of patients facing later lymph node or hematogenous metastases compared with those 
having a local relapse or no relapses at all $(P=0.048)$ (Figure 2a). Particularly later lymph node relapses were indicated by MMP-2 overexpression, $84 \%$ of cases with later lymph node relapses having a tumor with extensive or moderate MMP-2 positivity. A statistically significant correlation was found between MMP-2 immunoreactive protein overexpression and relapse-free survival. The cumulative 5year relapse-free survival rate for the patients with negative or weak positivity for MMP-2 was $61 \%$, as opposed to $42 \%$ for those with moderate or extensive staining of MMP-2 $(P=0.045$, in Breslow analysis) (Figure 3a). Patients with a MMP-2 negative or only weakly stained tumor survived longer than those with a tumor showing a moderate or extensive MMP-2 staining. The Kaplan-Meier analysis showed that the cause-specific cumulative
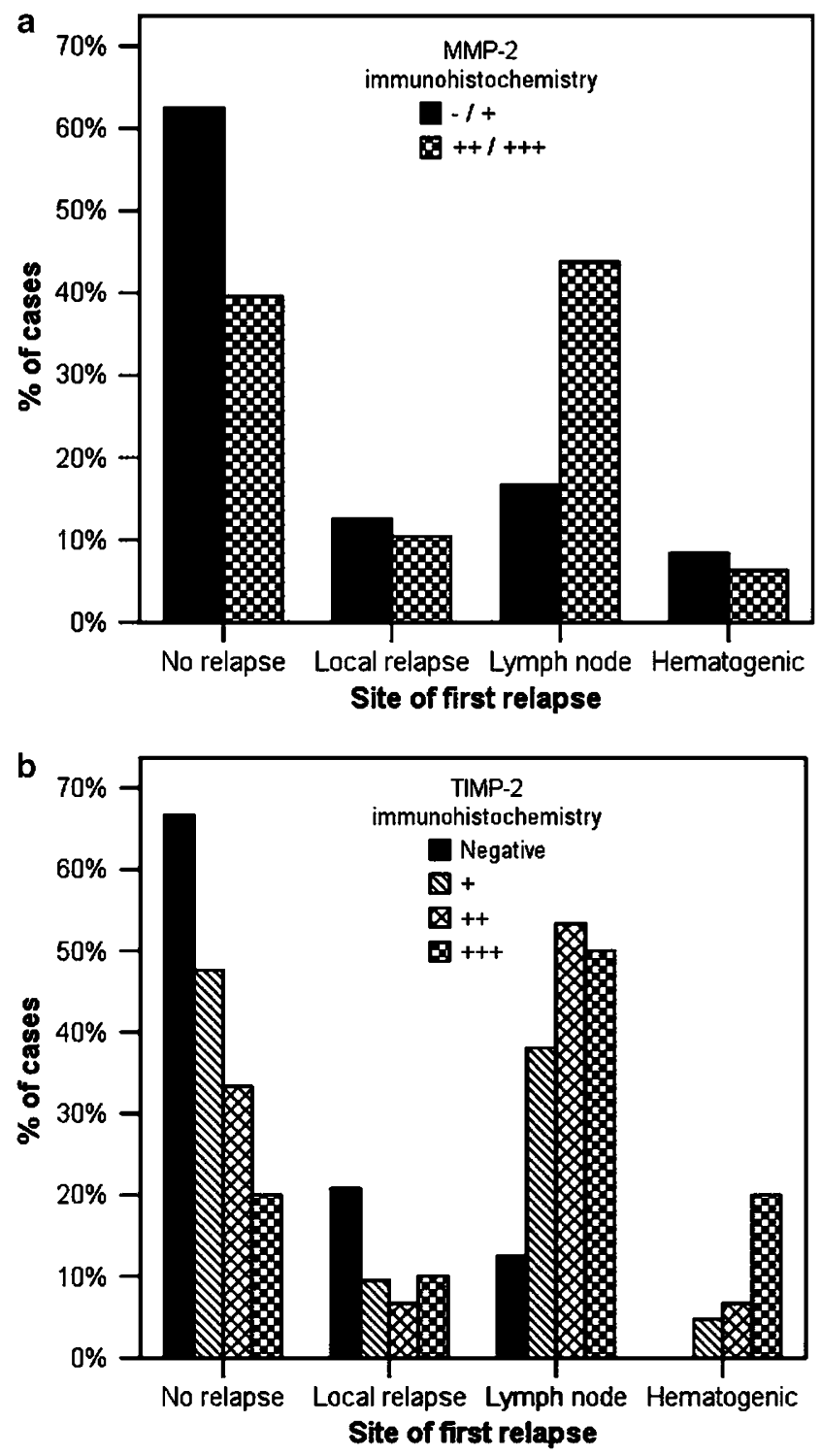

Figure 2 First site of relapse according the MMP-2 (a) and TIMP-2 (b) immunoreactive protein levels in primary tumor tissue. survival of the patients with negative or weak immunostaining was $66 \%$ after 5 years of followup, whereas the survival was only $46 \%$ among
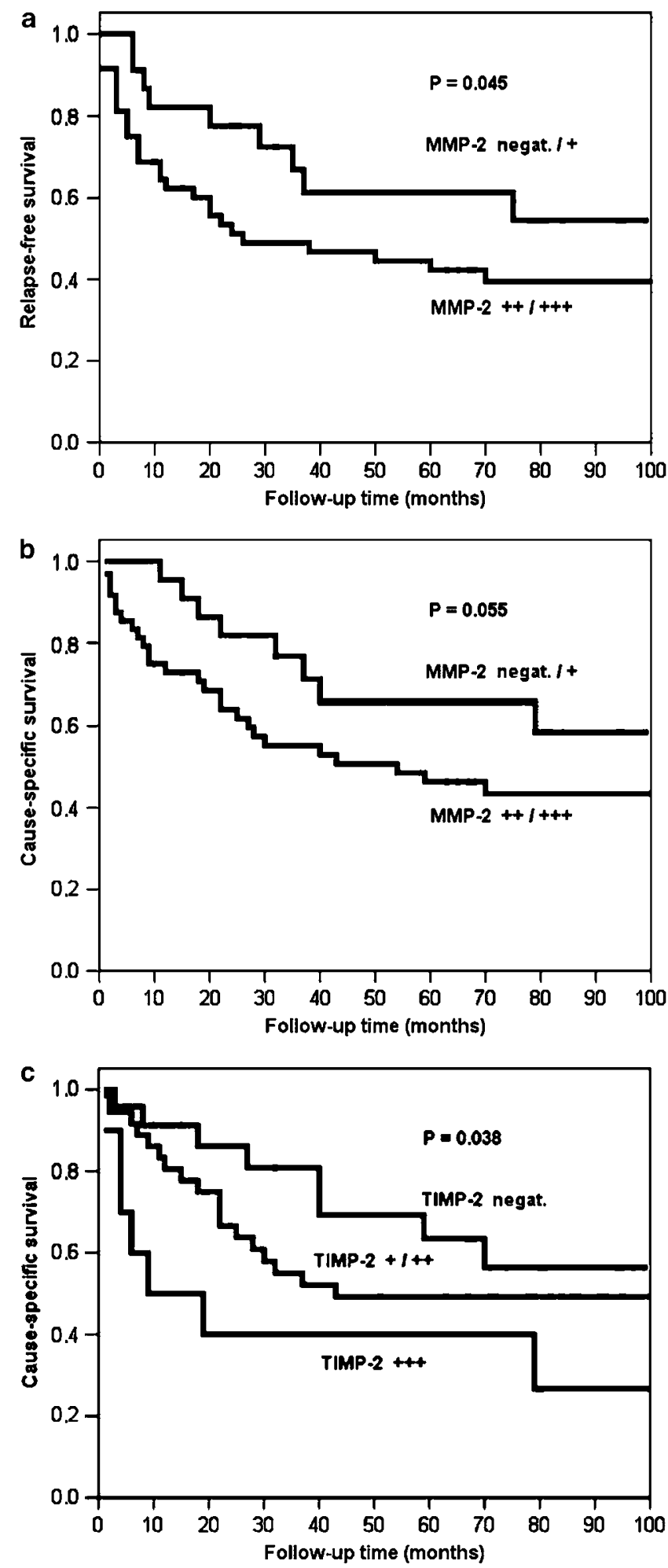

Figure 3 Survival analysis (Kaplan-Meier) for patients with HNSCC according to overexpression of tumor tissue MMP-2 (a and b) and TIMP-2 (c) immunoreactive protein. (a) Relapse-free survival (b) cause-specific survival and (c) cause-specific survival. 
the patients presenting with moderate or extensive immunostaining ( $P=0.055$, Breslow analysis) (Figure 3b).

\section{TIMP-2 as a Prognostic Factor}

Analogous to findings with MMP-2, also TIMP-2 was very strong indicator of later lymph node or hematogenous metastases. In all, $88 \%$ of local relapses appeared in cases with a TIMP-2-negative primary tumor, whereas $70 \%$ of cases with later lymph node or hematogenous metastases had strong positivity in TIMP-2 immunostaining $(P=0.01$, Fisher's exact test) (Figure 2b).

The survival analysis showed a statistically significant correlation between tumor immunoreactive protein expression of TIMP-2 and cause-specific survival. The Kaplan-Meier analysis showed that cumulative survival of patients with a TIMP-2negative tumor was $64 \%$ after 5 years of follow-up, whereas the cumulative survival for patients with a TIMP-2-positive tumor was $47 \%$. Furthermore we noticed that the cumulative 5 -year survival rate was the lowest among patients with a tumor showing extensive TIMP-2 immunostaining (40\%) (Figure 3c). This remarkable difference was also statistically significant $(P=0.038$, Breslow analysis). Positive TIMP-2 immunostaining also associated with shorter relapse-free survival, but the difference did not quite reach statistical significance in the log-rank analysis $(P=0.071)$. The cumulative 5 -year relapsefree survival rate was $60 \%$ in patients presenting with a negative staining for TIMP-2, whereas in patients with a positive TIMP-2 immunoreaction in the primary tumor the rate was only $43 \%$.

\section{Discussion}

Increased proteolytic activity is essential for the growth of tumor cells and their dissemination. There is a growing amount of clinical evidence that MMP-2 cascade promotes tumor cell invasion and the process of metastasis formation in several tumor types. MMP-2 has also been correlated to the clinical aggressiveness in some cancer types. ${ }^{9-11}$ HNSCC tumors have the capacity to invade adjacent tissues and metastasize locoregionally. Moreover, hematogenic relapses are possible, but more uncommon compared to some other solid tumors. Among HNSCC patients the major cause of death is expressly the locoregional recurrence, and the prognosis depends mostly on the stage of the tumor. ${ }^{2}$ However, the prognosis may also fluctuate greatly in each stage group, indicating that there is variation in biological behavior among this tumor group.

In this study we demonstrate that tumor tissue MMP-2 and TIMP-2 immunoreactivity associate with the biological aggressiveness of the HNSCC tumors and the clinical course of these diseases. The analyses in this study showed that $70 \%$ of the patients with later lymph node or hematogenic relapses had extensively positive TIMP-2 immunostaining in the primary tumor, indicating that more aggressive relapses were linked with TIMP-2 positivity. This correlation could be clinically noteworthy and it was statistically significant. Interestingly, MMP-2 overexpression was also significantly linked with aggressive relapses, particularly with the occurrence of later lymph node metastasis.

In the present study, it was also found that high expression of MMP-2 and TIMP-2 in tumor tissue predicts poor survival, supporting the theory that these markers could be linked with more aggressive behavior of the tumor. The MMP-2 immunoreactive protein associates highly with poor relapse-free survival as well as with cause-specific survival, 5year cumulative survival rates being 42 and $46 \%$ in patients with tumors presenting with an overexpression of MMP-2, when they were 61 and $66 \%$ in patients with low positivity of MMP-2 immunoreactive protein. Similarly, only $40 \%$ of the patients with an extensive TIMP-2-positive tumor survived for 5 years after the diagnosis of HNSCC, whereas in patients with a TIMP-2-negative tumor a $64 \%$ causespecific 5-year survival rate was observed. Accordingly, a difference in relapse-free survival was observed between patient groups presenting with positive or negative TIMP-2 immunoreactivity in the primary tumor. Our results are supported by some previous studies which have shown that high expression or activity of MMP-2 or TIMP-2 are linked with poorer survival in HNSCC. ${ }^{16-18,26-28}$ Interestingly, in those studies the prognostic role of both MMP-2 and TIMP-2 has been evaluated, and in each individual study either one of them has been found to be linked with prognosis in most cases. Actually, these studies have revealed somewhat conflicting results as to which one, MMP-2 or TIMP-2, is most important as a prognostic marker. In our present study we are able to show in same patient material that both of these immunoreactive proteins in tumor tissue are prognostic indicators for survival. In line with our study, Yoshizaki et $a l^{18}$ have reported that both MMP-2 and TIMP-2 are poor indicators in tongue SCC. Moreover, our results indicating more aggressive relapses linked with TIMP-2 expression are in line with Katayma et $a{ }^{27}{ }^{27}$ who have earlier reported that patients who developed regional lymph node and/or distant metastasis showed significantly higher scores in the expression of TIMP-2. Nevertheless, they found no correlation between later relapses and MMP-2 expression. Interestingly, in our study, we could not detect a significant correlation between the level of positivity with MMP-2 and TIMP-2 antibody staining and the traditional clinical or histopathological prognostic factors at the time of diagnosis. The lack of this correlation and the fact that MMP-2 and TIMP-2 are linked with later aggressive behavior of the disease support in particular the essential role of 
these markers as indicators of unfavorable clinical course. These data contradict some previous results but are in line with those of Katayama et $a l^{27}$ as well as with very recent results by Liu et al. ${ }^{28}$

In this study, we could not detect a correlation between the pretreatment circulating level of TIMP2, MMP-2:TIMP-2 complex or MMP-2 and the tumor tissue expression of immunoreactive proteins of MMP-2 and TIMP-2. Furthermore, no correlation was noticed between circulating levels of these immunoreactive proteins and relapse-free or causespecific survival. In the present study the mean levels of MMP-2:TIMP-2 complex were lower in HNSCC patients than in the serum of healthy controls. The same trend was observed in the level of circulating MMP-2 values. Moreover, the mean values of TIMP-2 serum levels were found to be nearly equal in HNSCC patients and healthy controls. Our data are supported by two previous studies, where no differences were found between serum MMP-2 levels in HNSCC patients and healthy controls. ${ }^{29,30}$ These data are also partly in line with a previous study by Ylisirniö et al, who reported that levels of TIMP-2 and MMP-2:TIMP-2 complex were higher among healthy controls compared with lung cancer patients, and no correlation with survival was observed..$^{23}$ On the contrary, postoperative serum MMP-2 has been previously reported to correlate with poor prognosis in breast cancer as well as to predict a recurrence of urothelial and colorectal carcinoma. ${ }^{31-33}$ Also serum MMP-2 has been reported with advanced stage in some type of cancers. ${ }^{34,35}$ Circulating TIMP-2 levels have been found to be very variable among cancer patients, and there is no clear consensus of its prognostic value in general.

It is known that many different types of tissues express continuously MMP-2, and it takes part in many physiologic tissue-remodeling processes. Moreover, unlike MMP-9, MMP-2 activity has only modest regulation at transcriptional level; most of the regulation occurs at post-transcriptional level and during activation and inactivation of the enzyme. ${ }^{3}$ It is also possible that the MMP-2 derived from tumor is not reflected in the serum in equal ratio. It is not clear why the results concerning the prognostic effect of circulating MMP-2 and TIMP-2 are so contradictory and unclear. One possible explanation is that the type of measured MMP-2 differs in each individual assay. In our study the assay recognizes the free pro-MMP-2 and that complexed with TIMP-2. This is in harmony with the immunostaining method we have used. Additionally, when the serum sample has been taken, there could be critical issues concerning differencies in the sample handling procedures that might modify the levels of circulating immunoreactive proteins.

The role of TIMP-2 in the process of tumor growth and invasion is somewhat unclear. Traditionally TIMP-2 has been supposed to suppress tumor invasion and metastatic cascade by inhibiting MMP-2. ${ }^{7-8}$ However, in the process of cell-mediated MMP-2 activation, TIMP-2 has been shown to have a crucial role together with MT1-MMP. ${ }^{36}$ Actually, TIMP-2 binding to pro-MMP-2 is mandatory for MTMMP binding and activation of pro-MMP-2. On the other hand, it has been shown that TIMP-2 stimulates normal and malignant cell growth in vitro and there is some evidence that this process is mediated by escalating the quantity of tyrosin phosphorylation and the activity of MAP kinase. ${ }^{37}$ Additionally, there are data supporting the notion that TIMP-2 could bind to the cell surface with high affinity, supporting the idea that TIMP-2 could work as a ligand equally to some growth factors and cytokines. $^{38}$ Separately from these roles of TIMP-2, it also seems to have antiapoptotic as well as antiangiogenic activity. ${ }^{7-8}$ As a matter of fact, the current literature indicates that the role of TIMP-2 in cancer cell growth and progression is extremely complex and may even differ between different cell types.

In conclusion, in the present study we found the tumor tissue expression of MMP-2 and TIMP-2 to be an indicator of unfavorable prognosis in HNSCC patients. However, the expression of MMP-2 and TIMP-2 immunoreactive protein did not correlate with the other clinical prognostic factors like the stage of the disease, the TNM status or the histological grade of the tumor, suggesting that MMP-2 and TIMP-2 could be independent from these factors. The pretreatment circulating levels of MMP-2, TIMP-2 and MMP-2:TIMP-2 complex did not, however, emerge as having any prognostic role in this study indicating that tumor tissue immunoreactive protein is more valuable as a prognostic factor. Supplementary studies are obviously needed to establish the role of MMP-2 and TIMP-2 as immunohistochemical markers in clinical use.

\section{Acknowledgements}

This study was supported in part by a grant from the Cancer Society of Northern Finland, Ida Montin Foundation and the Finnish Cancer Society. The authors thank Mrs Kaisu Järvenpää, Anne Bisi and Merja Matilainen for their skillful technical assistance during this work, as well as Mr Risto Bloigu for statistical consulting and the staff of the Otorhinolaryngology clinic at Oulu University Hospital for their help in collecting the serum samples.

\section{References}

1 Sanderson RJ, Ironside JA. Squamous cell carcinomas of the head and neck. Br Med J 2002;325:822-827.

2 Pivot X, Niyikiza C, Poissonnet G, et al. Clinical prognostic factors for patients with recurrent head and neck cancer: Implications for randomized trials. Oncology 2001;61:197-204. 
3 Nagase H, Woessner Jr JF. Matrix metalloproteinases. J Biol Chem 1999;274:21491-21494.

4 Nelson AR, Fingleton B, Rothenberg ML, et al. Matrix metalloproteinases: biologic activity and clinical implications. J Clin Oncol 2000;18:1135-1149.

5 Stetler-Stevenson WG, Yu AE. Proteases in invasion: matrix metalloproteinases. Semin Cancer Biol 2001;11: 143-152.

6 Stetler-Stevenson WG. The role of matrix metalloproteinase in tumor invasion, metastasis, and angiogenesis. Surg Oncol Clin N Am 2001;10:383-392.

7 Gomez DE, Alonso DF, Yoshiji $\mathrm{H}$, et al. Tissue inhibitors of metalloproteinases: structure, regulation and biological functions. Eur J Cell Biol 1997;74: 111-122.

8 Fassina G, Ferrari N, Brigati C, et al. Tissue inhibitors of matrix metalloproteinases: regulation and biological activities. Clin Exp Metastasis 2000;18:111-120.

9 Väisänen A, Kallioinen M, Taskinen PJ, et al. Prognostic value of MMP-2 immunoreactive protein (72 kD type IV collagenase) in primary skin melanoma. J Pathol 1998;186:51-58.

10 Talvensaari-Mattila A, Pääkkö P, Höyhtyä M, et al. Matrix metalloproteinase-2 immunoreactive protein: a marker of aggressiveness in breast carcinoma. Cancer 1998;83:1153-1162.

11 Kallakury BV, Karikehalli S, Haholu A, et al. Increased expression of matrix metalloproteinases 2 and 9 and tissue inhibitors of metalloproteinases 1 and 2 correlate with poor prognostic variables in renal cell carcinoma. Clin Cancer Res 2001;7:3113-3119.

12 Kawamata $\mathrm{H}$, Uchida $\mathrm{D}$, Hamano $\mathrm{H}$, et al. ActiveMMP2 in cancer cell nests of oral cancer patients: correlation with lymph node metastasis. Int J Oncol 1998;13:699-704.

13 Kurahara S, Shinohara M, Ikebe T, et al. Expression of MMPs, MT-MMP, and TIMPs in squamous cell carcinoma of the oral cavity: correlations with tumor invasion and metastasis. Head Neck 1999;21:627-638.

14 O-charoenrat P, Rhys-Evans PH, Eccles SA. Expression of matrix metalloproteinases and their inhibitors correlates with invasion and metastasis in squamous cell carcinoma of the head and neck. Arch Otolaryngol Head Neck Surg 2001;127:813-820.

15 Hong SD, Hong SP, Lee JI, et al. Expression of matrix metalloproteinase-2 and -9 in oral squamous cell carcinomas with regard to the metastatic potential. Oral Oncol 2000;36:207-213.

16 Yorioka CW, Coletta RD, Alves F, et al. Matrix metalloproteinase-2 and -9 activities correlate with the disease-free survival of oral squamous cell carcinoma patients. Int J Oncol 2002;20:189-194.

17 Ondruschka C, Buhtz P, Motsch C, et al. Prognostic value of MMP-2, -9 and TIMP-1, -2 immunoreactive protein at the invasive front in advanced head and neck squamous cell carcinomas. Pathol Res Pract 2002;198:509-515.

18 Yoshizaki T, Maruyama Y, Sato H, et al. Expression of tissue inhibitor of matrix metalloproteinase-2 correlates with activation of matrix metalloproteinase- 2 and predicts poor prognosis in tongue squamous cell carcinoma. Int J Cancer 2001;95:44-50.

19 Ruokolainen H, Pääkkö P, Turpeenniemi-Hujanen T. Expression of matrix metalloproteinase-9 in head and neck squamous cell carcinoma: a potential marker for prognosis. Clin Cancer Res 2004;10: 3110-3116.
20 Ruokolainen H, Pääkkö P, Turpeenniemi-Hujanen T. Serum matrix metalloproteinase-9 in head and neck squamous cell carcinoma is a prognostic marker. Int J Cancer 2005;116:422-427.

21 Ruokolainen H, Pääkkö P, Turpeenniemi-Hujanen T. Tissue inhibitor of matrix metalloproteinase-1 is prognostic in head and neck squamous cell carcinoma: comparison of the circulating and tissue immunoreactive protein. Clin Cancer Res 2005;11:3257-3264.

22 International Union Against Cancer. TNM Classification of Malignant Tumors. Wiley-Liss: Geneva, Switzerland, 1997, pp 7-49.

23 Shanmugaratnam K. In collaboration with Sobin LH and Pathologists in 8 countries. Histological Typing of Tumours of the Upper Respiratory Tract and Ear, 2nd edn. World Health Organization International Histological Classification of Tumours. Springer-Verlag: Berlin, 1991, pp 7-49.

24 Ylisirniö S, Höyhtyä M, Turpeenniemi-Hujanen T. Serum matrix metalloproteinases $-2,-9$ and tissue inhibitors of metalloproteinases $-1,-2$ in lung cancer- TIMP-1 as a prognostic marker. Anticancer Res 2000;20:1311-1316.

25 Kaplan EL, Meier P. Nonparametric estimation from incomplete observations. J Am Stat Assoc 1958;53: $457-481$.

26 Danilewicz M, Sikorska B, Wagrowska-Danilewicz M. Prognostic significance of the immunoexpression of matrix metalloproteinase MMP-2 and its inhibitor TIMP-2 in laryngeal cancer. Med Sci Monit 2003;9: $42-47$.

27 Katayama A, Bandoh N, Kishibe K, et al. Expressions of matrix metalloproteinases in early-stage oral squamous cell carcinoma as predictive indicators for tumor metastases and prognosis. Clin Cancer Res 2004;10: 634-640.

28 Liu WW, Zeng ZY, Wu QL, et al. Overexpression of MMP-2 in laryngeal squamous cell carcinoma: A potential indicator for poor prognosis. Otolaryngol Head Neck Surg 2005;132:395-400.

29 Riedel F, Gotte K, Schwalb J, et al. Serum levels of matrix metalloproteinase-2 and -9 in patients with head and neck squamous cell carcinoma. Anticancer Res 2000;20:3045-3049.

30 Kuropkat C, Plehn S, Herz U, et al. Tumor marker potential of serum matrix metalloproteinases in patients with head and neck cancer. Anticancer Res 2002;22:2221-2228.

31 Leppä S, Saarto T, Vehmanen L, et al. A high serum matrix metalloproteinase-2 level is associated with an adverse prognosis in node-positive breast carcinoma. Clin Cancer Res 2004;10:1057-1063.

32 Gohji K, Fujimoto N, Komiyama T, et al. Elevation of serum levels of matrix metalloproteinase- 2 and -3 as new predictors of recurrence in patients with urothelial carcinoma. Cancer 1996;78:2379-2387.

33 Oberg A, Höyhtyä M, Tavelin B, et al. Limited value of preoperative serum analyses of matrix metalloproteinases (MMP-2, MMP-9) and tissue inhibitors of matrix metalloproteinases (TIMP-1, TIMP-2) in colorectal cancer. Anticancer Res 2000;20:1085-1091.

34 Garbisa S, Scacgliotti G, Masiero L, et al. Correlation of serum metalloproteinase levels with lung cancer metastasis and response to the therapy. Cancer Res 1992;52:4548-4549.

35 Miyata Y, Kanda S, Nomata K, et al. Expression of metalloproteinase-2, metalloproteinase-9 and tissue inhibitor of metalloproteinase-1 in transitional cell 
carcinoma of upper urinary tract: correlation with tumor stage and survival. Urology 2004;63:602-608.

36 Shofunda K, Moriyama K, Nishihashi A, et al. Role of tissue inhibitor of metalloproteinase-2 (TIMP-2) in regulation of pro-gelatinase $\mathrm{A}$ activation catalyzed by membrane-type matrix metalloproteinase-1 (MT1MMP) in human cancer cells. Biochemistry 1998;124: $462-470$.
37 Yamashita K, Suzuki M, Iwata H, et al. Tyrosine phosphorylation is crucial for growth signaling by tissue inhibitors of metalloproteinases (TIMP-1 and TIMP-2). FEBS Lett 1996;396:103-107.

38 Hayakawa T, Yamashita K, Ohuchi E, et al. Cell growth promoting activity of tissue inhibitor of metalloproteinase-2 (TIMP-2). J Cell Sci 1994;107: 2373-2379. 